\title{
A Gathering of Native American Healers: Exploring the Interface of Indigenous Tradition and Professional Practice
}

\author{
Virgil D. Moorehead Jr. ${ }^{1} \cdot$ Joseph P. Gone ${ }^{2} \cdot$ Damia December $^{2}$
}

Published online: 8 September 2015

(c) Society for Community Research and Action 2015

\begin{abstract}
This article reports insights from a 4-day Gathering of Native American Healers at the University of Michigan in October of 2010. This event convened 18 traditional healers, clinically trained service providers, and cross-cultural mental health researchers for a structured group dialogue to advance professional knowledge about the integration of Indigenous healing practices and conventional mental health treatments in community-based mental health services for Native Americans. Our thematic analysis of transcripts from five Roundtable sessions afforded several key insights and understandings pertaining to the integration of Indigenous healing and conventional mental health services. First, with reference to traditional healing, the importance of a rampant relationality, various personal qualities, Indigenous spirituality, and maintenance of traditional life and culture were accentuated by Roundtable participants. Second, for traditional healers to practice effectively, Roundtable participants posited that these individuals must maintain personal wellness, cultivate profound knowledge of healing practices, recognize the intrinsic healing potential within all human beings, and work for the community rather than themselves. In speaking to the possibilities and challenges of collaboration between Indigenous and conventional biomedical therapeutic approaches, Roundtable participants recommended the implementation of cultural programming, the
\end{abstract}

Virgil D. Moorehead Jr.

mvirgil@stanford.edu

1 Counseling and Psychological Services, Vaden Health Center, Stanford University, 866 Campus Drive, Stanford, CA 94305, USA

2 Department of Psychology, University of Michigan, Ann Arbor, MI, USA observance of mutuality and respect, the importance of clear and honest communication, and the need for awareness of cultural differences as unique challenges that must be collaboratively overcome.

Keywords Native Americans - Traditional healing · Multicultural counseling · Mental health services . Alternative medicine

\section{Introduction}

Contemporary Native Americans are the descendants of the Indigenous peoples of the United States and Canada, including about 2.5 million American Indian or Alaska Native individuals in the US, and perhaps 1.5 million First Nations, Inuit, and Metis individuals in Canada (Canadian Census Bureau 2011; for an overview, see Gone and Trimble 2012). In the long wake of European colonization, Native American reservation and urban communities continue to suffer from pronounced mental health disparities in both national contexts, and the respective federal governments retain responsibility for addressing the mental health needs of these communities through the provision and/or funding of behavioral health services. Additionally, biomedical health services have become the primary institutional means by which Native American people obtain behavioral health care, in spite of the concurrent revival of Indigenous healing practices within these communities that has occurred over the past four decades (Gone 2010; Mohatt et al. 2004; Waldram 2014). In fact, even as locally based, culturally sensitive approaches to working with Native clients with mental health needs have emerged, psychosocial distress within tribal communities is typically managed in accordance with the stock 
conventions of the "psy" disciplines (e.g., psychology, psychiatry, social work) in on- and off-reservation behavioral health clinics (Gone and Trimble 2012).

As one might expect, great variety exists between and within the several hundred tribal communities in the US and Canada owing to both remarkable linguistic and cultural diversity prior to European contact, as well as long and unique histories of interaction with European settlers and their descendants in an increasingly globalized world. What most unites these diverse populations is the legacy of European (and, later, Euro-American and Euro-Canadian) dispossession, denigration, conquest, and control. The social distress experienced by Native American peoples is well documented, with recent estimates revealing epidemic levels of psychiatric illness exceeding the rates of other ethnoracial groups (IHS 2014). Researchers and practitioners working with Native populations theorize that the impact of structural violence on Indigenous communitieslately designated as historical trauma (i.e., the impacts of collectively experienced mass trauma passed from ancestors to descendants) (Brave Heart 2003; Duran 2006; Gone 2014) — has resulted in a range of current pathologies, including heightened levels of substance dependence, posttraumatic stress, interpersonal violence, youth behavioral problems, and suicide (Beals et al. 2005a; Centers for Disease Control and Prevention 2012).

\section{Diverse Therapeutic Traditions}

Despite these difficulties, Native American communities have exhibited uncommon resilience in the face of what might have been considered insurmountable obstacles. Indeed, beginning largely in the 1970s, contemporary Native people have pursued Indigenous cultural reclamation and revitalization projects at unprecedented rates (Allen et al. 2014; Fisher and Ball 2003; LaFromboise and Howard-Pitney 1995; Rasmus et al. 2014), which, in many respects, reflects a shift beyond mere survival to actual thriving. An important component of this Indigenous cultural revitalization is an emergent social movement dedicated to the reclamation and promotion of Native healing. Champions of these Indigenous therapeutic traditions have emphasized the reclaiming and adapting of traditional social relations, Indigenous knowledge, and ceremonial practices long disrupted, denigrated, and suppressed through colonization (Adelson 2000; Duran and Duran 1995; Waldram 2004). In doing so, Native Americans have increasingly expressed their power to act in their own interests, grounded in a shared belief that healing from historical injustices and continuing inequalities might be found through Indigenous cultural practices. Specifically, participation in traditional healing practices is thought to strengthen cultural identity, bolster community support systems, and promote political empowerment, all of which have been recognized as potential hallmarks of resilience for Indigenous communities (Chandler and Lalonde 1998, 2008; Kirmayer et al. 2011).

And yet, even as this continent's First Peoples have increased in population while confronting demoralizing political and economic conditions with creativity, fortitude, and resilience, the current outlook for reducing mental health disparities in Native American communities is not promising. Research shows that the majority of Native people with mental health needs do not obtain formal treatment (Beals et al. 2005b; Sue 1977). Moreover, complications frequently emerge for those who do seek assistance, in part due to the fact that mental health services delivered specifically for these communities are largely dependent on the resources and actions of the respective federal government. For example, in the United States, an estimated $55 \%$ of Native Americans rely on the Indian Health Service (IHS) for their health care (IHS 2006), and yet the socioeconomic and geographical barriers to obtaining available services have been well documented (IHS 2006, 2014). Moreover, IHS funding for mental health services is extremely limited. Recent per capita expenditures for personal health care for the IHS user population were just $\$ 2741$ in comparison to $\$ 6809$ for the general US population (IHS 2011), with only $10 \%$ of the funds allotted for clinical services within the IHS being dedicated to mental health treatment.

Beyond these challenges for remedying Native American mental health disparities lies an additional important obstacle, namely, significant cultural differences surrounding the therapeutic endeavor. For example, as medical anthropologists have long demonstrated, the experience and expression of illness is culturally configured. More specifically, cultural norms, understandings, and expectations govern (often implicit) concepts of wellness and dysfunction, and, thereby, contribute to the experience and expression of mental health problems, as well as strategies of coping, help seeking, and recovery (Gone and Kirmayer 2010). These culturally constituted beliefs and practices can be remarkably durable, persisting even in novel or hybrid forms in the wake of cross-cultural contact in ways that continue to differentiate people between and within particular communities. Interestingly, professional practices in mental health services are often presented as if they were the transcendent, culture-free products of objective observation and scientific inquiry (Kirmayer 2007); and yet, there are no such things as culture-free professional practices, leading to the realization that the conventions of the psy-disciplines (e.g., psychotherapy) are themselves cultural technologies or artifacts. Such conventions can be problematic with respect to Indigenous community mental health owing to unfamiliar cultural sensibilities 
surrounding the therapeutic endeavor. For example, Gone (2010) delineated three key differences between the principles and practices of professional psychosocial treatment and Indigenous traditional healing, with the former privileging secular, rational, and technical approaches to intervention while the latter emphasizes the sacred, mysterious, and relational aspects of therapeutic process.

\section{Integration of Therapeutic Traditions}

Nevertheless, over the past several decades, mental health professionals and Indigenous traditional healers have sought to integrate or incorporate Indigenous healing practices into formal mental health services in an effort to maximize scarce resources, legitimate Indigenous therapeutic traditions, and render mainstream psychosocial interventions more accessible to Native American clients. One example of such attempts can be found in a 1973 issue of the American Journal of Psychiatry, in which psychiatrist Robert Bergman described his routine interactions with Navajo healers in a locally run "school for medicine men." This article is unusual, however, in the level of detail it provides about such collaborations, as other literature of this type rarely includes nuanced description of the many conceivable issues surrounding collaboration between traditional healers and mental health service providers within Native community health care systems (for an overview, see Gone 2010). As one means of remedying this scholarly oversight, the second author organized a 4-day Gathering of Native American Healers at the University of Michigan in October of 2010. This event convened 18 traditional healers, clinically trained service providers, and cross-cultural mental health researchers for a public exchange about the interface of Indigenous healing practices and mental health treatments in community-based services for Native Americans.

More specifically, the purpose of the Gathering was to convene representatives of these three constituencies to engage in a structured group dialogue to advance professional knowledge about the integration of Indigenous healing practices and conventional mental health treatments in community-based services for Native Americans. The participants were invited as small teams of individuals comprised of Indigenous traditional healers (THs), Native American service providers (SPs), and both Native and non-Native mental health researchers (MHRs) with histories of prior collaboration in diverse regions of the US and Canada (from Detroit, Michigan; Albuquerque and the nearby Navajo reservation in New Mexico; the Blackfeet Indian reservation in Montana; and Saskatoon in the Canadian province of Saskatchewan, respectively). The entire group met in retreat for 2 days at the Fetzer Institute in Kalamazoo, MI, to collectively prepare for a culminating 1.5-day Roundtable on Native American Culture, Gender, and Healing at the University of Michigan that was dedicated to a public exploration of these issues. During the retreat portion of the Gathering, participants opened each day with a prayer and ceremony, introduced themselves to one another at length, and then undertook a process resulting in a consensual formulation of the structure for the public Roundtable. Importantly, participants agreed that explicit attention to both gender issues and community diversity throughout the Roundtable was desirable. At the end of the retreat, participants subsequently relocated to Ann Arbor for the public event with a clear sense of group purpose, Roundtable structure, presentation topics, and speaking assignments.

The Roundtable was held on October 14-15, 2010, in the School of Social Work at the University of Michigan. The structure of the Roundtable deliberately borrowed from a common sequence in ceremonial protocol, namely, an opening session, four "rounds" devoted to consideration of specific content, and a closing session. The Opening Session was comprised of an introduction to the Roundtable, including its origins and purpose. Round One was dedicated to introducing the four teams and describing their experience with culture and treatment in four Native American communities. Round Two was dedicated to considering the question, What is traditional healing? Round Three was dedicated to considering the question, Who is an effective traditional healer? Round Four was dedicated to considering the question, What are the possibilities for collaboration? The Closing Session afforded summary reflections. The Opening and Closing Sessions featured the MHRs as the presenters; these individuals also served as moderators for each round. Each round was scheduled for $105 \mathrm{~min}$ and featured a presenter from each of the four teams (as designated by their teams), with all team members presenting in at least one of the four rounds. The order of presentation for each round was also inspired by ceremonial protocol, beginning with the designated speaker from the Detroit team and followed by representatives from each of the New Mexico, Blackfeet, and Saskatchewan teams, respectively, in rough correspondence to the four cardinal directions (east, south, west, and north). Thus, each round consisted of four presentations, followed by a moderated dialogue in response to these presentations among the Roundtable participants themselves, and concluding with a facilitated exchange between participants and the audience. The Roundtable was recorded so that lessons drawn from this utterly distinctive event might be analyzed, summarized, and disseminated to a wider scholarly audience, which is the purpose of this article. 


\section{Method}

\section{Participants}

The Detroit team consisted of individuals associated with the urban American Indian health organization in this region known as American Indian Health and Family Services, Inc. Heading the Detroit team was Jerilyn LeBeau-Church (Lakota), the director of the organization and a clinically trained social worker. Detroit team members included Mona Stonefish (Pottawtamie/Mohawk), a traditional healer and cultural advisor to the health center; Anthony Davis (Odawa), the cultural counselor from the behavioral health program at the center; and Dr. Sandra Momper (Ojibwe), a professor of Social Work at the University of Michigan who served as both research partner and board member at the center.

Heading the team from New Mexico was Chenoa Bah Stillwell-Jensen (Diné), the community outreach coordinator for a large children's health initiative in that state. Team members included David Johns (Diné), a traditional healer and artist; and Lorenzo Jim (Diné), a substance abuse counselor who utilized both conventional and traditional treatment modalities in the urban setting of Albuquerque. The New Mexico team did not include a previously affiliated MHR.

Heading the team from the Montana was Patrick Calf Looking (Blackfeet), the longtime director of the Blackfeet Nation's residential substance abuse treatment program known as Crystal Creek Lodge. Team members included Lyena Fish (Blackfeet), a substance abuse counselor and cultural authority; Richard Ground (Blackfeet), a traditional healer and bundle keeper for the traditionalist Crazy Dog Society; and Larry Ground (Blackfeet), a facilitator for the Foundation for Historical Grief and Trauma and also a traditional leader for the Crazy Dog Society. The second author, Dr. Joseph P. Gone (Gros Ventre), a cultural-clinical psychologist on faculty at the University of Michigan (and organizer of the Gathering), also participated as the MHR for this team.

Heading the team from the First Nations University of Canada in Saskatchewan was Dr. Richard Katz, professor emeritus at First Nations University in Saskatchewan, an author of three books on Indigenous healing whose recent work included longstanding collaborations with Aboriginal people in Canada. Team members included Mary Lee (Plains Cree), a cultural advisor and youth worker with expertise in traditional parenting and women's teachings; Danny Musqua (Saulteaux), a ceremonial leader and cultural advisor to the Indian Social Work program at the university; and Tania LaFontaine (Métis), a social worker by training who taught at the university while drawing on her clinical expertise with troubled Aboriginal youth.

Two additional participants in the Roundtable included Lyle Noisy Hawk, Jr. (Lakota), a youth worker and doctoral candidate in counseling psychology with distinctive expertise in bridging psychotherapy and healing; and Dr. Laurence Kirmayer, a cultural psychiatrist who directs Canada's National Network for Aboriginal Mental Health Research as well as the Division of Social and Transcultural Psychiatry at McGill University. Additional biographical information about all conference participants is available on request from the authors.

\section{Transcripts}

All Roundtable sessions were recorded and transcribed for analysis, except for the Opening Session, as it consisted of a formal, structured presentation by the organizer rather than a series of presentations followed by dialogue and audience exchange. Initial transcription was conducted by a clerical services company with no knowledge of Indigenous traditional healing or mental health professional practices. Thus, the transcripts were subsequently reviewed and corrected by a Native American alumnus of the University of Michigan who attended the Roundtable and maintained familarity with and interest in these issues. Finally, an undergraduate summer research intern finalized the transcripts, offering a handful of minor corrections and formatting changes. Consequently, the data analyzed for this article include the corpus of finalized transcripts for five sessions of the Roundtable, comprising about 80-single spaced pages of transcript.

\section{Procedure}

In order to systematically identify the principal lessons of the Roundtable for purposes of understanding the complexities surrounding integration of Indigenous traditional healing and mental health services, a thematic analysis (Braun and Clarke 2006) was conducted with the transcripts from the five Roundtable sessions. Thematic analysis is typically adopted for "identifying, analyzing, and reporting patterns (themes) within data" (p. 79), frequently with a goal of interpreting the meaning and significance of such patterns for some knowledge domain. A key quality of thematic analysis is its flexibility in application for analyzing a wide range of qualitative data. In this instance, our straightforward task was to distill the public presentations and responses of 18 different participants-in interaction with each other and an audience across five sessions devoted to unpacking distinct but related questions-into 
shared understandings and insights (i.e., themes) pertaining to the issue of therapeutic integration. Thus, our analysis exploits the flexibility of this approach to apply it to an utterly distinctive event (without precedent, so far as we know). Nevertheless, the following analytic process remains recognizable as a clear instance of thematic analysis (specifically, as a theoretical thematic analysis, according to Braun and Clarke 2006), and follows an accessible logic that, far from being mystifying, should instead seem relatively intuitive to a broad scholarly audience. For purposes of clarity, we will illustrate this process with intermittent examples.

The analysis commenced with the third author, a senior undergraduate research assistant at the time, working closely with her faculty mentor (the second author) to determine a suitable strategy for thematic analysis of the Roundtable transcripts. Once settled, all coders subsequently received careful guidance and feedback from the second author throughout the analytic process. The subsequent analysis proceeded in six distinctive steps. First, the third author decomposed the transcript of each round into separate sub-transcripts comprised of the material from each individual participant who spoke at any time during the round (e.g., ten sub-transcripts were created for Round Two, that is, for each of the participants who said something). This ensured that basic ideas were initially generated from within participants (rather than across participants) as well as within sessions (rather than across sessions), so as to preserve the meaning and coherence of individual contributions offered in response to specific session-based questions. Second, the third author distilled the content of each round's sub-transcript in sequential fashion to further reduce the content of each speaker's contribution to comprehensive but succinct summary ideas. To accomplish this, she subdivided all text within each speaker's sub-transcript by major idea, and then provided a condensed statement for every idea (or related set of ideas) expressed throughout the text.

For example, participant David Johns presented for about 12 min during Round Two, and his presentation was subdivided into 13 separate ideas. These ideas were distilled from two single-spaced pages of sub-transcript to 13 summary statements requiring only one-half of one page. For example, David Johns stated during his presentation:

The natural elements plays a major role to healing. The Creator and the holy people provided these elements for specific reason and purpose. The songs and prayers coincide with the natural elements. Without the proper protocol, the prayers, along with the songs, the natural elements, and the spirits of those elements are not at their full power. The sacredness of elements rings true and strong. Our reliance on natural elements are vital to the healing process. The traditional healing takes place where healing is with one's determination to be healthy.

In contrast to these 90 words, the third author condensed this related set of ideas to three shorter sentences: "The natural elements (songs, prayers, and holy sites) were provided by the Creator and relying on them is vital to the healing process. Without the proper protocol (ceremony), these elements are not at their full power. Traditional healing occurs when one is determined to be healthy."

Third, once all transcribed material had been summarized in this fashion, the third author proceeded to extract and collate ideas (i.e., codes, the basic element of a thematic analysis) reflecting the ideational content of the summary statements of all participants throughout the Roundtable. She entered these into a comprehensive spreadsheet for each session that listed every code derived from participant statements within the session, including a tally of all participants who endorsed each of the derived codes. For example, the spreadsheet for Round Two lists 42 codes in total as expressed by the ten participants who spoke during this session. Eighteen codes were expressed by David Johns during his presentation, including six derived (at least in part) from the quotation in the previous paragraph: healing as a spiritual process, prayer as integral to healing process, nature as central to identity, ceremonies heal, relying on natural elements, and determination to be healthy. Moreover, the code healing as a spiritual process was also expressed by three other participants in this round (Gone, L. Ground, and Davis). Beyond this, coded expressions of basic ideas by any participant from any other session were also recorded in the spreadsheet in which the code initially appeared. For example, for the coded entry for healing as a spiritual process in the Round Two spreadsheet, two other expressions of this code (Davis and Musqua) were noted from the Round One sub-transcripts. In total, 160 codes were recorded across all presenters in the five sessions. Endorsement of coded ideas ranged from an absolute frequency of 1-7 instances per code and from 1 to 6 participants who expressed any given coded idea (reflecting the fact that some presenters repeated their ideas across sessions).

Fourth, a check on the validity of this coding strategy was undertaken separately by the first author, who received his doctoral degree in psychology in the midst of data analysis. In close consultation with the second author as a faculty mentor to ensure continuity of approach across coders, the first author confirmed the derivation of the 160 codes, but identified and corrected five instances in which participants mentioned a basic idea that the initial coder did not capture, and four instances in which a code was tallied 
that had not actually been expressed. Fifth, in the wake of this validation process, the first author created a Master Code spreadsheet that listed all coded ideas and all instances of endorsement across the 18 Roundtable participants, ranked within sessions by number of participants to express a coded idea (in descending order). This Master Code spreadsheet (available from the authors upon request) represented the final corpus of data that formed the basis for subsequent consolidation and selection of interpreted themes for inclusion in this article.

Sixth, and finally, while attending to both the frequency of coded ideas, as well as the number of participants to endorse a given idea, the first and second authors collaborated on the interpretive generation of themes (i.e., patterned responses in the data that are selectively judged as important relative to the original purpose of the analysis) in light of the orienting questions that structured each session of the Roundtable. This was an admittedly subjective process in certain respects, which, according to Braun and Clarke (2006), is inescapable: "An account of themes 'emerging' or being 'discovered' is a passive account of the process of analysis, and it denies the active role the researcher always plays [italics added] in identifying patterns/themes, selecting which are of interest, and reporting them to the readers" (p. 80). Thus, it is the interpretive warrant for formulating our findings that matters in the selection and generation of themes, which is why we have detailed our analytic procedure in such detail. In sum, we have identified, reviewed, defined, and named the themes on the basis of the Master Code spreadsheet as reported below in accordance with Braun and Clarke's step-by-step description. Every effort was made to adhere to their 15 criteria for "good thematic analysis" (p. 96).

\section{Results}

In this study, Roundtable participants considered three primary questions at the interface of traditional healing and mental health services within Indigenous communities: (a) What is traditional healing?, (b) Who is an effective traditional healer? and (c) What are the possibilities for collaboration across these disparate therapeutic traditions? In response to each of these questions, four themes were generated from the coded ideas presented by Roundtable participants across sessions. Each of the 12 themes was endorsed by between four and ten out of the 18 participants in the Roundtable.

\section{What is Traditional Healing?}

The importance of relationships to all manifested as the most common theme in defining effective traditional healing practices. Nine participants in Round Two, plus one participant in Round Three, strongly advanced a belief that traditional knowledge and practice are fundamentally dependent on relationships with others, and that these relationships are believed to extend well beyond a dyadic patient-healer relationship to include relations with the Creator, family, community, and the world in general. One participant, Mona Stonefish (TH, Detroit team), seemed to speak for the majority by maintaining that, "to promote healing, we have to understand that it is necessary for a renewal of relationships," and we must "embrace [each other] because we are all interconnected, all related." Five participants specified that the restoration and "maintenance of balance and harmony" among all sacred Powers, human beings, and the natural environment can be strongly unified with traditional healing. In other words, traditional healing promotes harmony and a feeling of unity with all "the natural elements, songs, holy sites, supreme creator, and oneself," and that all these entities live in relationship to each other. Accordingly, six participants noted the profound power of kindness and love to all living and nonliving entities as essential to traditional healing, further highlighting the strong relational thread that runs through most descriptions of traditional healing practices.

For seven participants during Round Two, one participant during Round Three, and one participant during Round Four, the importance of personal qualities, such as "faith and belief" in traditional healing, "self-discipline," "treating the body well," and "laughter," was considered essential to effective traditional healing practices. David Johns (TH, New Mexico team) captured several of these sentiments when he stated,

Healing begin[s] with a competent and psychological decision to get back into balance. Traditional healing is available to all who need it, [however], the faith and belief in traditional healing is needed for the healing process to begin and to be successful. The true intent of the desire to be healthy and happy lies in the healing process.

Other participants' echoed John's views maintaining that altruism, "self-sacrifice," "respect for oneself and others," and the overall "determination to be healthy" are fundamental to traditional healing.

The importance of spirituality was the third theme that we noted in our analysis. Here, the contours of Indigenous knowledge and healing practices were described by five participants during Round Two and one participant during Round Three as inextricably spiritual in nature, in the sense that they are shaped by sacred orders of space and time. Two participants emphasized that the spirit world exists side-by-side with the physical world while simultaneously intermingling with it. Five participants communicated that 
within Indigenous spiritual processes, "prayer and ceremonies are integral," frequently functioning as elementary components of traditional healing. Included in this emphasis on spirituality was the belief that, within Indigenous communities, participation in spiritual ceremonies and other tribal specific ritual traditions is of primary importance. Using the Pikuni (Southern Piegan or Blackfeet) peoples' sun lodge as an example, Larry Ground (TH, Blackfeet team) discussed the Creator's lodges (and ceremonies used therein) as "beacon[s]" for the people of the Blackfeet nation, and spaces that bring "sanctuary, love, and happiness," while serving to create "balance" in the community. Ground explained that within these lodges people contribute their time and energy to pray for the Creator to hear them and assist in healing. According to him, the sun lodges are the "holiest places" for the Pikuni people, with ample evidence of "miracles happening." These miracles occur within a spiritual space, similar to other Indigenous healing practices.

Five participants during Roundtable Two and one during Round Three identified the importance of maintaining traditional life and culture as a fourth central theme in traditional healing practices. Indeed, whether speaking of Navajo, Blackfeet, Mohawk, or other First Nations cultural traditions, participants believed traditional healing to be rooted in Indigenous histories and cultural teachings. In fact, four participants emphasized that, within their tribal cultures, "tradition is healing." The importance of teaching these traditional ways of healing was evident throughout all four panel discussions. To this end, Mary Lee (TH, Saskatchewan team) stated that she was taught the traditional ways of Cree life, such as being educated by her mother on the "tepee teachings," which she now passes on to her grandchildren. Lee stated,

As a woman, the teachings are very powerful in my family, in my community. Women are the center of the fire as we were named with that fire in the center of that tepee. In my language we [are] called Iskweyo (women).

Lee emphasized that that these teachings, obtained from her mother, stress that women bring discipline, warmth, and comfort to their communities and, as part of this process, provide guidance for future generations. For Lee and other participants, these kinds of traditional teachings-especially the vital roles that women play in traditional life - are essential to traditional healing.

\section{Who is an Effective Traditional Healer?}

During Round Three, eight participants spoke to the characteristics of an effective healer. Perhaps the most striking and prominent feature of the participants' description of an effective traditional healer was the importance placed on the wellness of the healer. Five participants during Round Three and two participants during Round Two placed considerable emphasis on the emotional and psychological health of the traditional practitioner. Danny Musqua (TH, Saskatchewan team) stated that a healer must embody traditional teachings, be "fearless," and "stand on their virtues." Other participants evinced that a traditional healer should "teach by example," exemplify individual traits such as "humility and trustworthiness," and consistently act with integrity. For instance, Lyena Fish (SP, Blackfeet Team) asserted, "I always believe that we have this inner spirit within us, and whatever we put into our bodies, take into our bodies, will make that spirit sick. So, that's why it's so important to begin to heal yourself." This inclination toward personal wellness also included the necessity of self-awareness, or knowing "who you are," and drawing power from oneself. Here, participants stressed that becoming personally well can be a life-long endeavor, and is a process that transpires largely through traditional healers' own participation in healing ceremonies.

Accordingly, acquiring the knowledge of traditional healing practices - including ritual mediation, ceremonial supplication, and associated tribal traditions-was another qualification that seven participants during Round Three, one participant during Round Two, and one participant during Round Four listed for effective traditional healers. Regarding healers within these Indigenous traditions, two participants stated that attaining this knowledge occurs through a lengthy apprenticeship in which a traditional healer learns the nuances of spiritual practice and the ways of diagnosis and treatment of individual dysfunction. Healers must learn the "meaning of medicine" from another established elder or healer in the community and then find someone to whom that could pass that knowledge. For instance, David Johns (TH, New Mexico team) provided a concrete example of the ways in which a traditional healer gains legitimacy through an arduous apprenticeship. As a member of the Hitahili Association, a group of healers from the Navajo Nation, Johns stated that his traditional apprenticeship spanned 20-30 years. During this time, he learned complex ceremonies, how to assemble a sacred medicine bundle, and ways to build relationships with the community. For Johns, this process involved intense personal growth through learning the knowledge of healing within his Navajo tradition.

While participants underscored the role traditional healers actively play in acquiring knowledge and becoming healthy, five participants placed additional emphasis on the intrinsic potential for healing that all persons possess. For example, three participants stressed that all people have the ability to "heal themselves." Mona Stonefish (TH, Detroit 
team) stated that "everyone has some sort of medicine." Lorenzo Jim (SP, New Mexico team) asserted, "each individual, clan, family unit or member possesses a traditional healing quality." Three participants thought a major role of an effective traditional healer was to assist people in walking their intended path. In other words, healers help others to heal themselves.

The last theme describing effective traditional healers regards the belief that traditional healers do not work for themselves, but instead work for the community. Three participants during Round Three and one during Round Two discussed the ways in which traditional healers are recognized and then chosen by the community to serve these roles. For Anthony Davis (SP, Detroit team), a healer is chosen because of:

The way they carry themselves in the community, the way they carry the culture. The way they use the language. A healer is used to help that community to be whole. A healer is chosen because [of] what they do and how they interact in the community.

In addition, Davis stated that this selection process is based on the scope of their efficacy, such as if their interactions heal the entire community. Regarding the healer's relationship with the community, four participants stated that healers must place the interests of the community before their own. The importance of the healer's responsibility and duty to the community makes their selection a particularly serious matter. Indeed, three participants stated that the primary role of many traditional healers is not so much "treatment" for "mental health problems" as much as "healing" toward "collective wellness."

\section{What are the Possibilities for Collaboration?}

In discussing possibilities and challenges at the interface of Indigenous approaches to healing and mental health services, 11 participants offered numerous recommendations. Collaboration through cultural programming appeared as the most frequently suggested method to achieve such pivotal goals, with five participants during Round Four and one during Round One believing it to be vital. Chenoa Bah Stillwell-Jensen (SP, New Mexico team) described this process as moving from being "passive recipients of the system" to proactively working together to build more culturally sensitive systems. Jerilyn LeBeau-Church (SP, Detroit team) described her role in developing cultural programs in an urban American Indian health care setting in Detroit. LeBeau-Church stated that when she first became director of American Indian Health and Family Services (AIHFS), it quickly became apparent that the community wanted traditional healing practices to be utilized. To start, because of the prerequisite to accentuate evidence-based practices when requesting funds and additional programming from government officials, she knew that assessments had to be completed. Thus, she assisted in implementing focus groups with community members to assess for the need for cultural programs. She also brought two Lakota healers to facilitate conversations around bringing traditional healing services to the community. Additionally, she recommended that every staff member examine their own spiritual growth, path, and practice. She then introduced smudging, prayer, and talking circles throughout the services and activities at AIFHS. By virtue of this process, AIHFS built an on-site sweat lodge available to members of the community. For LeBeau-Church, "spirituality is now at the core of the work done at AIHFS." In brief, LeBeau-Church's example provides a useful picture of the possibilities of implementing cultural programming while working within the parameters of a biomedically based health care model.

Four participants during Round Four, one during Round Three, and one during Round One also discussed the significance of mutuality and respect in creating workable collaborations. Here, the necessity of epistemic egalitarianism (or equally valuing knowledge from the two therapeutic traditions) was seen as imperative. For instance, Richard Ground (TH, Blackfeet team) stressed that is essential to "treat everybody in the world with respect" and "they'll treat you with respect." For Ground, "this is our teaching that we have to do." Similarly, Richard Katz (MHR, Saskatchewan team) stressed that there is always collaboration, interplay, or a mutual interface when two people have contact. However, the important question becomes whether these interactions occur in equitable fashion. For Katz, true collaboration "really does not have a hierarchical value system that one is better than the other, one is primary to the other, [or] one is before the other." Rather, true collaboration relies on equality. Katz provided an example of this process by discussing how an elder with whom he once closely worked told him that "only a fool would resist surgery. But let's prepare for surgery with a ceremony." Katz provided this example to highlight the importance of respecting all healing traditions; both surgery and ceremony should be treated equally, and, by doing so, a principle of "synergy" can ensue in which ceremony can make surgeries even more effective. Significantly, two participants stated that during such collaborations it is essential that Indigenous ceremonies be implemented carefully and by those who know what they are doing.

As part of this call for respect and mutuality, five participants placed a strong emphasis on clear and honest communication. For example, Tania LaFontaine (SP, Saskatchewan team) asserted her experience that collaboration is "all about relationships." To have effective relationships, clear communication is essential. LaFontaine 
described her work in a 24-h-a-day government lock-down institution for kids between ages 12 and 17, emphasizing the ways in which she learned to communicate in order to promote collaboration with co-workers. Over the years, she reported that she developed open and honest communication strategies with her employers as the key to trust. Trust was developed in large part by being honest about who she was, and communicating this to her colleagues. As such, LaFontaine reported a sense of collegial respect toward her as a result of effective communication, leading to a "situation that works beautifully."

As much as participants seemed hopeful that cultural programming, respectful relationships, and clear communication could help pave the road to effective collaboration, participants were also well aware of the challenges to achieving this collaboration. Five participants during Round Four and one participant during Round Two mentioned the ways in which cultural differences can lead to challenges in attaining such collaboration. Among the cultural factors that may detract from collaboration is the necessity placed on scientific evidence to show the efficacy for mental health programs. More specifically, two participants stressed that the emphasis within psychology on the superiority of "positivistic, materialistic, and quantitative data" can be a barrier to implementing culturally sensitive interventions within those Indigenous communities that may not value this particular way of knowing. Similarly, the difficulties around adapting "cultural knowledge to modern life" and maintaining "cultural integrity" during collaborations were noted as other significant cultural barriers. The diverse traditions of Indigenous communities and varying value systems of these distinct cultural groups were also listed as a potential barrier. To this end, Patrick Calf Looking (SP, Blackfeet team) recommended that learning to "integrate all services from all entities, with representatives from all tribes, is a must."

\section{Summary}

Analysis of the Roundtable sessions afforded several key insights and understandings pertaining to the integration of Indigenous healing and mental health services. First, with reference to traditional healing, the importance of a rampant relationality, various personal qualities, Indigenous spirituality, and maintenance of traditional life and culture were accentuated by Roundtable participants. Second, for traditional healers to practice effectively, participants posited that traditional healers must maintain personal wellness, cultivate profound knowledge of traditional healing practices, recognize the intrinsic healing potential within all human beings, and work not for themselves but for the community. In speaking to the possibilities and challenges of collaborating between biomedical and
Indigenous therapeutic approaches, Roundtable participants recommended collaboration through cultural programming, the observance of mutuality and respect, the importance of clear and honest communication, and the need to look out for cultural differences that might lead to new and difficult challenges.

\section{Discussion}

Gone (2010) delineated important differences between Indigenous healing traditions and biomedical technologies such as psychotherapy (e.g., sacred vs. secular therapeutic orientations) that present formidable obstacles to implementing effective mental health approaches for Native North American communities (see also, Goodkind et al. 2010; Lafromboise et al. 1990; Trimble et al. 2014). While it is not our intention to proliferate unconstructive dichotomies by framing our discussion in terms of an Indigenous/Western opposition, our point of departure must necessarily reflect the mindsets of both mainstream mental health professionals as well as Native North American community members, including participants in the Roundtable. This dichotomy-Indigenous versus Western-emerged in the context of European colonization when newly arrived Europeans settlers promoted a "savagery" versus "civilization" discourse with reference to Native peoples (Deloria 1995). Centuries of subjugation, dispossession, and other colonial tactics have given rise to contemporary Natives sensitivities surrounding the validity and appeal of long-subjugated Indigenous cultural practices vis-a-vis frequently imposed Euro-American ideals and institutions. In the context of ongoing inequality, discrimination, and marginalization, many Native community members today in both the US and Canada continue to express interest in-and even preference for-markedly Indigenous supports and services (Beals et al. 2005a, b; Walls et al. 2006).

Despite the call by many within diverse Native communities for adoption and promotion of Indigenous practices within mental health services - and as different as biomedical and Indigenous approaches may be-the results of this analysis demonstrate that integrating Indigenous and biomedical healing practices may be particularly advantageous for addressing the unique health disparities that affect these communities. In certain respects, the optimism about therapeutic integration reflected in the words of Roundtable participants should not be surprising, as Indigenous people have engaged in creative acts of cultural consolidation since their initial contact with European settlers, producing numerous blended, fused, or hybrid cultural forms that have served adaptive purposes (e.g., BigFoot and Schmidt 2010). As a consequence, today's 
potential for integration among biomedical and Indigenous therapeutic approaches and activities remains promising. And yet, the politics of post-coloniality present ongoing challenges to therapeutic integration that will require thoughtful collaboration and pragmatic persistence.

\section{Relationships and the Integrative Endeavor}

Shared Indigenous experiences of European colonization created contemporary circumstances of entrenched inequality, disadvantage, and absence of opportunity that continue to derail Native American aspirations and prospects (Kirmayer et al. 2014; West et al. 2012). Such social, political, and economic realities have not only resulted in formidable Indigenous health disparities, but also in bitter legacies of community alienation, resentment, and demoralization relative to the dominant societies of the US and Canada. Thus, it seems fitting that the importance of relationships was articulated frequently in the Roundtable as being central to traditional healing and, by extension, a crucial and necessary element in any attempt at integration of mainstream and Indigenous therapeutic approaches. Indeed, it would seem that a variety of positive relational dynamics, such as kinship, self-compassion, gender respect, and a desire for harmony with all entities, provides the figurative threads that can weave distinct mental health approaches together. As these results suggest, cultural difference, in and of itself, may not be the central barrier to collaboration. Rather, it would appear that the largest barrier to true integration is that Indigenous lifeways, cultural practices, interpersonal sensibilities, and "ways of knowing" are not valued equally to those of their non-Native counterparts. Accordingly, in order to make mental health services more inclusive, both Native and non-Native professionals need to first recognize that whether it is called psychotherapy or traditional healing, relational dynamic-including the pursuit of healthy relationships with oneself and others-is where healing occurs. Secondly, mental health professionals should acknowledge that exchanges between practitioners of hegemonic and marginal healing systems do not usually occur on equal footing, thereby requiring unusually skilled relational sensitivity throughout such interactions.

\section{Consolidation and the Integrative Endeavor}

If the politics of post-coloniality require unusual relational skills for engaging in fruitful collaborations toward therapeutic integration, they also require ideational dexterity and practical flexibility toward the creative consolidation of diverse therapeutic activities. More specifically, Native American communities have understandably grown protective of Indigenous cultural practices-and especially spiritual traditions - that have been preserved or revitalized in spite of Euro-American and Euro-Canadian efforts to eradicate them throughout the colonial encounter. Thus, it is not uncommon for traditional healers to remain quite reticent about their practice, and for them to worry that non-Native cultural hobbyists, religious enthusiasts, or "New Agers" will misappropriate these endangered traditions for inappropriate purposes. And yet, the integrative endeavor requires an Indigenous openness to characterization, explanation, and even demonstration of healing practices for purposes of finding common ground, exchanging therapeutic information, building collaborative models, or creating institutional partnerships. Beyond this openness to sharing such details, the integrative effort will additionally require judicious alterations, accommodations, re-packagings, or re-framings of elements or components from practitioners on both sides of the integrative encounter. For Indigenous practitioners in particular, however, the politics of post-coloniality can easily press them to pursue a "hard line" rather than a "big tent," occasioning a retreat from the integrative project. As we have already noted, however, Indigenous peoples have long engaged in complex patterns of adoption, adaptation, and rejection of various cultural elements in the creation of novel cultural forms, and most Indigenous practitioners are intimately familiar with the ways in which their own "traditional" practices reflect these histories of engagement (Gone 2007; Kahn et al. 1988). The important point, of course, is that any novel hybrid forms will need to be locally developed or ratified to ensure accountability and legitimacy.

\section{Collaboration and the Integrative Endeavor}

Successful therapeutic integration will benefit from sustained collaborations between people occupying roles similar to the teams we brought together for the Roundtable. It is rare enough in mental health services today to attend to Indigenous traditional healing at all, much less to engage in dynamic partnerships between THs, SPs, and MHRs. And yet, the sensitivities and nuances surrounding the interface of these disparate therapeutic traditions virtually require trusting and sustained collaborations for novel integrative efforts to develop, and for exemplars and insights drawn from these to enter scientific publication. Thus, additional forums in which such collaborations might be showcased would seem to promise fruitful contributions to both knowledge and practice in the mental health field. Undoubtedly, such collaborations will yield further insights into both similarities and differences between Indigenous healing and biomedical approaches to mental health challenges that harbor the potential to exert bidirectional influences. For example, Gone (2009; see also Gone and 
Calf Looking 2015) noted that the primary goal of such integrative projects within Indigenous communities may not be so much to find treatment for individual mental health problems as much as to heal the collective "social" problems (i.e., dis-orders, dis-eases) that may afflict entire Native American populations. Interestingly, this re-socialization of problems that are conventionally framed as psychological may prove emancipatory for non-Native recipients of mental health services as well.

\section{Limitations}

Certain limitations of our analysis should be considered. First, the participants in the Roundtable that provided the basis for this analysis represented small teams from four communities in the US and Canada, and cannot possibly reflect the full diversity and range of Native American communities. Accordingly, the results of this Roundtable discussion must be considered within its specific history, structure, and context. Yet, there is such limited research on the question of Indigenous and biomedical therapeutic integration that this utterly distinctive Gathering of Healers represents an important first step toward understanding the potential for further collaborations of this kind. Second, our analysis of the Roundtable discussion featured a tailored analytic procedure that probably has no precise precedent in the mental health literature. Nevertheless, we have attempted to describe the rationale for our analysis in accessible fashion and to demonstrate the rigor of our method for transcribing, analyzing, coding and interpreting the results in ways that we hope assure confidence in the dependability and credibility of our findings.

\section{Conclusion}

In this article, we reported insights from a 4-day Gathering of Native American Healers featuring traditional healers, clinically trained service providers, and cross-cultural mental health researchers who sought to advance professional knowledge about the integration of Indigenous healing practices and conventional mental health treatments in community-based mental health services for Native Americans. Participants in the Roundtable considered three primary questions at the interface of traditional healing and mental health services within Indigenous communities: (a) What is traditional healing?, (b) Who is an effective traditional healer? and (c) What are the possibilities for collaboration across these disparate therapeutic traditions? Our thematic analysis of transcripts from five Roundtable sessions afforded several key insights and understandings pertaining to the integration of Indigenous healing and mental health services. A major lesson from our study is that forums such as the Roundtable can afford unique opportunities for the exchange of knowledge, perspective, and practice that promise to enrich therapeutic integration efforts throughout Native America.

Acknowledgments The Healer's Roundtable was sponsored by the National Center for Institutional Diversity (NCID), Michigan Institute for Clinical and Health Research (MICHR), Office of the Provost, Department of Psychology, Native American Studies Program, and the School of Social Work at the University of Michigan, and by the Fetzer Institute. This article was completed during the second author's tenure as the Katz Family Endowed Chair in Native American Studies at Montana State University in Bozeman, MT. Additionally, the second author wishes to express his gratitude to the John Simon Guggenheim Memorial Foundation for fellowship support during the completion of this article.

\section{References}

Adelson, N. (2000). 'Being alive well': Health and the politics of Cree well-being. Toronto, ON: University of Toronto Press.

Allen, J., Mohatt, G. V., Beehler, S., \& Rowe, H. L. (2014). People awakening: Collaborative research to develop cultural strategies for prevention in community intervention. American Journal of Community Psychology, 54, 100-111.

Beals, J., Manson, S. M., Whitesell, N. R., Spicer, P., Novins, D. K., Mitchell, C. M., et al. (2005a). Prevalence of DSM-IV disorders and attendant help-seeking in 2 American Indian reservation populations. Archives of General Psychiatry, 62, 99-108.

Beals, J., Novins, D., Whitesell, N., Spicer, P., Mitchell, C., Manson, S., et al. (2005b). Prevalence of mental disorders and utilization of mental health services in two American Indian reservation populations: Mental health disparities in a national context. American Journal of Psychiatry, 162, 1723-1732.

Bergman, R. L. (1973). A school for medicine men. American Journal of Psychiatry, 130, 663-666.

Bigfoot, D. S., \& Schmidt, S. R. (2010). Honoring children, mending the circle: Cultural adaptation of trauma-focused cognitivebehavioral therapy for American Indian and Alaska Native children. Journal of Clinical Psychology, 66(8), 847-856.

Braun, V., \& Clarke, V. (2006). Using thematic analysis in psychology. Qualitative Research in Psychology, 3(2), 77-101.

Brave Heart, M. Y. H. (2003). The historical trauma response among Natives and its relationship with substance abuse: A Lakota illustration. Journal of Psychoactive Drugs, 35, 7-13.

Canadian Census Bureau. (2011). The aboriginal population profile. Retrieved from http://www12.statcan.gc.ca/nhs-enm/2011/dp$\mathrm{pd} /$ aprof/index.cfm?Lang=E\&fpv $=10000$.

Centers for Disease Control and Prevention, National Center for Injury Prevention and Control. (2012). Suicide: Facts at a glance. Retrieved from http://www.cdc.gov/ViolencePrevention/ pdf/Suicide_DataSheet-a.pdf.

Chandler, M. J., \& Lalonde, C. E. (1998). Cultural continuity as a hedge against suicide in Canada's First Nations. Transcultural Psychiatry, 35(2), 191-219.

Chandler, M. J., \& Lalonde, C. E. (2008). Cultural continuity as a protective factor against suicide in First Nations youth. Horizons, $10,68-72$.

Deloria, V. (1995). Red earth, white lies: Native Americans and the myth of scientific fact. Goldon, CO: Fulcrum Publishing.

Duran, E. (2006). Healing the soul wound: Counseling with American Indians and other Native people. New York, NY: Teachers College Press. 
Duran, E., \& Duran, B. (1995). Native American postcolonial psychology. Albany, NY: State University of New York.

Fisher, P. A., \& Ball, T. J. (2003). Tribal participatory research: Mechanisms of a collaborative model. American Journal of Community Psychology, 32(3-4), 207-216.

Gone, J. P. (2007). "We never was happy living like a Whiteman": Mental health disparities and the postcolonial predicament in American Indian communities. American Journal of Community Psychology, 40(3-4), 290-300.

Gone, J. P. (2009). A community-based treatment for Native American historical trauma: Prospects for evidence-based practice. Journal of Consulting and Clinical Psychology, 77(4), $751-762$.

Gone, J. P. (2010). Psychotherapy and traditional healing for American Indians: Exploring the prospects for therapeutic integration. The Counseling Psychologist, 38, 166-235.

Gone, J. P. (2014). Reconsidering American Indian historical trauma: Lessons from an early Gros Ventre war narrative. Transcultural Psychiatry, 51(3), 387-406.

Gone, J. P., \& Calf Looking, P. E. (2015). The Blackfeet culture camp: Auditioning an alternative indigenous treatment for substance use disorders. Psychological Services, 12(2), 83-91.

Gone, J. P., \& Kirmayer, L. J. (2010). On the wisdom of considering culture and context in psychopathology. In T. Millon, R. F. Krueger, \& E. Simmonsen (Eds.), Contemporary directions in psychopathology: Scientific foundations of DSM-V and ICD11 (pp. 72-96). New York, NY: Guilford Press.

Gone, J. P., \& Trimble, J. E. (2012). American Indian and Alaska Native mental health: Diverse perspectives on enduring disparities. Annual Review of Clinical Psychology, 8, 131-160.

Goodkind, J. R., Ross-Toledo, K., John, S., Hall, J. L., Ross, L., Freeland, L., et al. (2010). Promoting healing and restoring trust: Policy recommendations for improving behavioral health care for American Indian/Alaskan Native adolescents. American Journal of Community Psychology, 46(3-4), 386-394.

Indian Health Service. (2006). Facts on Indian Health Disparities. Rockville, MD: Indian Health Services. http://www.ihs.gov/ PublicAffairs/IHSBrochure/files/disparitiesfacts-Jan2006.pdf.

Indian Health Service. (2011). IHS year 2011 Profile, July 15. Rockville, MD: Indian Health Service. http://www.ihs.gov/ PublicAffairs/IHSBrochure/Profile2011.asp.

Indian Health Service. (2014). IHS Fact Sheets: Indian health disparities. Retrieved March 15, 2015, from http://www.ihs.gov/ newsroom/factsheets/disparities/.

Kahn, M. W., Jejero, L., Anton, M., Francisco, D., \& Manual, J. (1988). An indigenous community service on the Tohono
O'ondham (Papago) Indian reservation: Seventeen years later. American Journal of Community Psychology, 16(3), 369-379.

Kirmayer, L. J. (2007). Psychotherapy and the cultural concept of the person. Transcultural Psychiatry, 44(2), 232-257.

Kirmayer, L. J., Dandeneau, S., Marshall, E., Phillips, M. K., \& Williamson, K. J. (2011). Rethinking resilience from Indigenous perspectives. Canadian Journal of Psychiatry, 56(2), 84-91.

Kirmayer, L. J., Gone, J. P., \& Moses, J. (2014). Rethinking historical trauma. Transcultural Psychiatry, 51(3), 299-319.

LaFromboise, T. D., \& Howard-Pitney, B. (1995). The Zuni life skills development curriculum: Description and evaluation of a suicide prevention program. Journal of Counseling Psychology, 42(4), 479.

LaFromboise, T. D., Trimble, J. E., \& Mohatt, G. V. (1990). Counseling intervention and American Indian tradition: An integrative approach. The Counseling Psychologist, 18(4), $628-654$

Mohatt, G. V., Hazel, K. L., Allen, J., Stachelrodt, M., Hensel, C., \& Fath, R. (2004). Unheard Alaska: Culturally anchored participatory action research on sobriety with Alaska Natives. American Journal of Community Psychology, 15(3-4), 95-116.

Rasmus, S. M., Charles, B., \& Mohatt, G. V. (2014). Creating Qungasvik (a Yup'ik intervention "toolbox"): Case examples from a community-developed and culturally-driven intervention. American Journal of Community Psychology, 54(1-2), 140-152.

Sue, S. (1977). Community mental health services to minority groups: Some optimism, some pessimism. American Psychologist, 32(8), 616-624.

Trimble, J. E., King, J., Norman, D., BigFoot, D. S., \& LaFromboise, T. (2014). American Indian and Alaska Native Mental Health Perspectives. In R. Parekh (Ed.), The Massachusetts General Hospital textbook on diversity and cultural sensitivity in mental health (pp. 119-138). New York, NY: Springer. doi:10.1007/ 978-1-4614-8918-4.

Waldram, J. B. (2004). Revenge of the Windigo: The construction of the mind and mental health of North American Aboriginal peoples. Toronto, ON: Toronto Press.

Waldram, J. B. (2014). Healing history? Aboriginal healing, historical trauma, and personal responsibility. Transcultural Psychiatry, 51(3), 370-386

Walls, M. L., Johnson, K. D., Whitbeck, L. B., \& Hoyt, D. R. (2006). Mental health and substance abuse services preferences among American Indian people of the northern Midwest. Community Mental Health Journal, 42(6), 521-535.

West, A. E., Williams, E., Suzokovich, E., Strangeman, K., \& Novins, D. (2012). A mental health needs assessment of urban American Indian youth and families. American Journal of Community Psychology, 49(3-4), 441-453. 